1 Establishing wonder oil,

2 Solanesol, as a novel inhibitor for 3 Focal Adhesive Kinase by in silico ${ }_{4}$ strategies 
24 Betty Daneial ${ }^{1}$, Devashish Das ${ }^{2}$, Jacob Paul V.J ${ }^{1}$, Guruprasad R ${ }^{3 *}$

25 1. Department St Joseph's college, Bangalore, India.

26 2. Scientist, Educept, New Delhi, India

27 3. Scientific Director, Durga Femto Technologies and Research (DFTR), \#22, $4^{\text {th }}$ main, $4^{\text {th }}$ cross,

28 Chamrajpet, Bangalore-560018, India, drgp@dftr.org, Mo.+91-9880293053, Corresponding

29 Author

30 


\section{Establishing wonder oil, Solanesol, as a novel inhibitor for Focal Adhesive 32 Kinase by in silico strategies}

Focal adhesion kinase (FAK) plays a primary role in regulating the activity of many signaling molecules. Increased FAK expression has been implicated in a series of cellular processes, including cell migration and survival. Inhibiting the activity of FAK for cancer therapy is currently under investigation. Hence, FAK and its inhibitors has been the subject of intensive research. To understand the structural factors affecting inhibitory potency, kinetic analysis, molecular docking and molecular dynamics simulation were studied in this project. Though, Solanesol was found have inhibitory activities towards FAK, no in silico tests were ever done on the same.

Due to high flexibility of Solanesol (Rotatable bonds $=25$ ), it is difficult to analyze using normal docking protocols. This paper introduces a novel method to dock and analyze molecules with high flexibility based on weighed contact based scoring method. This method uses blind docking technique, which was developed for protein peptide docking method, to generate conformations which were used to calculate contact based weights of residues. This method reveals the possible binding site for the small molecule. An exhaustive docking search on the acquired area reveals the docked confirmation of the compound. The final docked conformation was subjected to molecular dynamics to understand of binding stability. This study is in a good agreement with experimental results which shows Solanesol binds at ATP binding site and inhibit the phosphorylation of Focal Adhesion Kinase. 


\section{Background}

\section{Focal Adhesion Kinase}

60 Among the 10 hallmarks of cancer, "Tissue Invasion and Metastasis", is the most devastating

61 one, as it allows the oncogenic cells to migrate to new areas in the body with more open space

62 and more nutrients. It significantly reduces survival rates and prognosis for patients. The loss of

63 cellular adhesion to the extracellular matrix regulation can lead to increased cellular

64 proliferation, decreased cell death, and altered cellular differentiation status and cellular

65 migratory capacity (Hanahan \& Weinberg, 2000; Hanahan \& Weinberg, 2011; Lazebnik, 2010;

66 van Nimwegen \& van de Water, 2007).

67 The FAK4 family kinases, including Focal Adhesion Kinase (FAK), regulate cell adhesion,

68 migration, and proliferation in a variety of cell types. Focal adhesions are heterodimeric-

69 transmembrane integrin receptors located within sites of close opposition to the underlying

70 matrix which mediate adhesion of cells to extracellular matrix. TYR397 position FAK

71 phosphorylation simulated by Integrin engagement and clustering creates high affinity binding

72 site for SRC and SRC family kinases. In turn, FAK-SRC complex phosphorylates many

73 components of focal adhesion, which result in changes in initiation of signaling cascades and

74 adhesion dynamics. Other than the FAK catalytic activity, FAK additionally functions as a

75 scaffold to organize signaling and structural proteins within focal adhesions (Cohen \& Guan,

76 2005; Guan, 1997).

77 Alteration in FAK expression not only have been associated with tumorigenesis and increased

78 metastatic potential, it is also reported to cause multiple cancers, including colon, breast, thyroid,

79 prostate, cervical, ovarian, head and neck, oral, liver, stomach, sarcoma, glioblastoma, and 
melanoma (Guan, 1997).

\section{Solanesol}

83 Solanesol is a polyisoprenoid alcohols or polyprenols, found mainly accumulates in solanaceous

84 crops, including tobacco, tomato, potato, eggplant, and pepper plants.In industries, Solanesol is

85 extracted commercially from it richest source, tobacco plant.Commercially, Solanesol is widely

86 used as an intermediate for the synthesis of ubiquinone drugs, such as coenzyme Q10 and

87 vitamin $\mathrm{K} 2$ as well as Vitamin $\mathrm{K}$ and Vitamin E. It known to possess activities like antibacterial,

88 antifungal, antiviral, anticancer, anti-inflammatory, and anti-ulcer activities, and its derivatives

89 also have anti-oxidant and anti-tumor activities, in additionto other bioactivities (Yan et al.,

90 2015; Srivastava et al, 2009; Suzuki, Tomida \& Nishimura, 1990; Tomida \& Suzuki, 1990;

91 Zhao, Zu, Li, \& Tian, 2007; Severson, Ellington, Schlotzhauer, Arrendale, \& Schepartz, 1977).

92 Derivatives of Solanesol, (S)-2,3-dihydropolyprenyl, monophosphate, and agents for inhibiting

93 the metastasis of cancers (Okamoto, Tsuji, \& Yamazaki, 1994).

\section{Objective}

95 The main aim of this paper is to establish Solanesol as a Focal adhesion kinase inhibitor by the

96 means of in silico methods. Though Solanesol used as bioactive agent in industries for decades,

97 due to its highly flexible nature, there is no successful in silico protein binding and simulation

98 data available online till date.

99 Solanesol has 25 rotatable bonds which makes very difficult to dock directly to the protein

100 structure by conventional method. Although there are various methods for prediction of binding 
101 site in FAK, the flexible nature of Solanesol as well as the size of the compound makes it

102 difficult to fit the active site. For the purpose of predicting the actual binding site, blind docking

103 method can be used. Blind docking method was introduced for the purpose of docking peptide

104 molecules to the protein molecule but is a tested method for binding of small molecules when

105 binding site is unknown (Hetényi \& van der Spoel, 2006).

106 Method of blind docking comprise of locking of ligand molecule's all the torsions, for the

107 purpose of reducing calculation time, and then docking on the complete protein surface. Then the

108 best cavity or binding pocket is selected, based on the clustering of highest binding affinity

109 conformations (Hetényi \& van der Spoel, 2006; Hetényi \& van der Spoel, 2002).

110 In this paper we propose a method of binding of highly flexible compound to protein targets with

111 using an enhanced contact based scoring method. This method scores the residues rather than the

112 conformations. The higher scored residues where then used for more "focused" docking on those

113 residue region.

\section{Materials and methods}

\section{Protein selection and preparation}

116 The crystallographic co-ordinates for Focal adhesion kinase (PDB ID: 4Q9S) (George et al,

117 2014) were retrieved from the Protein Data Bank (PDB). Prior to docking, protein structures

118 were prepared by removing water molecules using UCSF Chimera software (Pettersen et al,

119 2004). Following which, bond orders were assigned, and hydrogen atoms were added to the

120 crystal structures. 


\section{Ligand preparation}

122 Solanesol exist in both cis and trans states, for this experiment we considered only trans as it is

123 only found in natural sources (Roe, Oldfield, Geach, \& Baxter, 2013). The structure of Solanesol

124 was obtained from PubChem compound (CID 5477212) (Kim et al, 2015; NCBI 2016). Gaussian

12509 program (Frisch et al, 2009) was used to obtain the optimum geometry of the structures using

126 the density function theory at the B3LYP/6-31G (d,p) level.

\section{$127 \quad$ Molecular docking}

128 All the molecular docking studies of Solanesol to FAK were performed using Autodock 4.2

129 (Morris et al, 2009). Autodock uses a semi-empirical free energy force field to evaluate binding

130 conformations of ligand while docking. The AutoDockTools (Morris et al, 2009) was used for

131 preparing protein and ligand parameters files.

133 Binding site analysis:

134 Solanesol is a 45 carbon chain with 26 rotatable bonds. As it is extremely flexible it is hard to

135 determine the bind mode of it with the protein. The commonly used protocol for determination of

136 binding pocket is "Blind docking", which was initially developed for to determining peptide

137 docking with protein (Hetényi \& van der Spoel, 2006; Hetényi \& van der Spoel, 2002). In this

138 method the constrained ligand (or peptide) is docked with the whole protein surface. The place

139 where it forms a cluster with higher energy determines the binding site. Then these sites were

140 used for "refined docking" where the lowest binding modes for each of these places (in case if

141 there are more than one) where determined by molecular mechanics and molecular dynamics

142 studies. 
143 For calculating the possible area of interaction or binding site of a highly flexible ligand we

144 enhanced blind docking using Ligand Contact Based Scoring function for Residues (LCBSR).

145 This is an atomic contactlclash based scoring method, in which the residues are scored based on

146 the higher favorable interactions and probability of formation of a hydrogen bond. As it doesn't

147 depend on the clustering or solely on binding energy, it statistically enhances the probability of

148 finding the possible binding site.

149 It can be represented as,

$$
\operatorname{LBCSR}=\log \left(\mathrm{N}_{\mathrm{Co}}-\mathrm{N}_{\mathrm{Cl}}\right)-\log \left(\mathrm{N}_{\mathrm{H}} / \mathrm{C}_{\mathrm{Cl}}\right)
$$

151 Where, ligand contact based scoring of residue (LCBSR) is calculated using "Number of

152 Contacts" (NCo) which is the number of occurrences where atoms of residue (r) are in contact

153 with any atom of the ligand in all the conformations. "Number of Clashes" ( $\mathrm{NCl})$ is the number

154 of occurrences when atoms of residue (r) are in clashes or unfavorably overlapped with any atom

155 of the ligand in all the conformations. "Number of Hydrogen Bonds" (NH) is the number of

156 occurrences when atoms of residue (r) are forming a hydrogen bond with any atom of the ligand

157 in all the conformations. "Number of Clashes" $(\mathrm{CCl})$ is the number of conformers where residue

158 (r) is in an unfavorable overlap with any atom of the ligand.

159 The "Contact" here is defined as the instance when the difference between the distance of two

160 atoms and the sum of their van der Waal radii is $0.4 \mathrm{~A}$ or more or, in other words, the distance is

161 greater than the sum of van der Waal radii (Eq 1) of two atoms. Whereas "Clash" is defined as

162 the condition where the van der Waal radii of two atoms unfavourably overlaps each other and

163 the distance is lower than the sum of radii (Eq 2) of the two atoms. This can be represented as: 


$$
\Sigma \mathrm{r}_{\mathrm{VDW}}(\mathrm{i}, \mathrm{j})-\mathrm{D}_{\mathrm{ij}} \geq-0.4
$$

$$
\Sigma \mathrm{r}_{\mathrm{VDW}}(\mathrm{i}, \mathrm{j})-\mathrm{D}_{\mathrm{ij}} \geq 1.0
$$

166 Where, $\Sigma r_{V D W}(i, j)$ is the sum of van der Waal radii of interacting atoms of ligand (i) and residue

167 (j) and $D_{i j}$ is the distance between interacting atoms of ligand (i) and residue (j). A Higher value

168 of LCBSR of any residue implies the residue may be a part of the binding pocket for the ligand

169 and, if it is capable, it may also form a hydrogen bond with the ligand. Lesser score implies a

170 lower interaction or high chances of unfavorable clashes or low chances of forming a hydrogen

171 bond.

\section{Experimental design:}

\section{Blind docking}

174 For this experiment, Solanesol was docked a total of 3 times (Table 1) with "Blind Docking"

175 protocol. For getting an unbiased result, all the rotatable bonds were kept unconstrained for all

176 the experiments. The protein was covered using a $126 \times 126 \times 126$ grid box with protein centre

177 as grid centre. For experiment ligand starting position was changed.

178 The docking resultant file from Autodock was then converted into multiple PDB files using

179 Autodock scripts. All the contacts and clashes, as well as hydrogen bonds between the

180 conformation and protein, were calculated using UCSF Chimera tool.

181 Considering all the conformers may lead to false positives, thus conformers were separated in 182 three criteria:

183 (1) Binding energy less than $-2.0 \mathrm{kcal} / \mathrm{mol}$ 
(2) Binding energy less than $-3.0 \mathrm{kcal} / \mathrm{mol}$

(3) Binding energy less than $-4.0 \mathrm{kcal} / \mathrm{mol}$.

186 Provided -4.0 is roughly half the average of the binding energy (Average B.E $=-7.10 \mathrm{kCal} / \mathrm{mol}$ )

187 of all the three experiments, ensuring only conformations with low B.E were considered. The

188 resultant values from each of these three were added to corresponding residues. This way the

189 residues interacting more with the conformations of better binding energy will have a better

190 score.

191 For getting a statistically significant result, all the scores of the residues from all the three

192 experiments were added to get the final score of each residue. Only those residues which

193 appeared in more than 2 experiments were considered.

\section{Refined docking}

195 A binding site was formed using residues with a higher LCBSR score (percentile $=0.50$ ). This

196 binding site was then used for refined docking using Autodock. The experiment was done twice

197 with (1) relaxed parameters, GA maximum energy evaluations $2.5 \times 106$, for 200 GA runs (2)

198 exhaustive parameters, GA maximum energy evaluations $3.5 \times 107$, for 200 GA runs.

\section{Knowledge-based rescoring}

200 All conformations were rescored using DSX, Drug Score eXtended, (Neudert \& Klebe, 2011),

201 knowledge-based rescoring based on the DrugScore formalism, to estimate the affinity of

202 conformation for FAK. The best conformations were selected based on the rescored values. The

203 best conformation bound complex of FAK was further used for molecular dynamics simulation

204 studies. 


\section{Molecular dynamics}

Molecular dynamics simulations for FAK protein as well as Solanesol bound FAK were

207 performed using the GROMACS (Groningen Machine for Chemical Simulations) 4.6 (Hess,

208 Kutzner, Van Der Spoel, \& Lindahl, 2008) software with GROMOS96 (53a6) force field.

209 PRODRG (Van Aalten et al, 1996) server was used to generate topology files for Solanesol.

210 Charges were kept full and no energy minimization was done using PRODRG.

211 The complex was solvated in a dodecahedron box with SPC model water model

212 molecules and periodic boundary conditions were used. One negatively charged chlorine ion $(\mathrm{Cl}-$

213 ) was added to the system for maintaining the system's neutrality.

214 The Lincs and Shake algorithm (Hess, Bekker, Berendsen, \& Fraaije, 1997) were used

215 for constraining bond length and fixing all bonds containing hydrogen atoms respectively.

216 For electrostatic calculations, Particle Mesh Ewald (PME) (Darden, York, \& Pedersen,

217 1993) method was used, with a coulomb cutoff of $1.2 \mathrm{~nm}$, Fourier spacing of $0.16 \mathrm{~nm}$ and an

218 interpolation of order 4. Energy minimization of the system was carried out using steepest

219 descent algorithm with a tolerance value of $1000 \mathrm{~kJ}$ mol-1nm-1. After energy minimization,

220 NVT and NPT equilibrations were done on the system until it reached the room temperature and

221 water density. Production MD was performed for $20 \mathrm{~ns}$ time duration for both the simulations.

\section{Molecular dynamics trajectories analysis}

223 Root mean square deviation (RMSD) and root mean square fluctuations (RMSF) of FAK

224 backbone were calculated using "g_rms" and "g_rmsf” utility commands, respectively. A

225 spherical probe of radius $1.4 \AA$ across the protein surface was used for calculating solvent-

226 accessible surface area (SASA) by "g_sasa" tool of Gromacs. Hydrogen bonds between 
227 Solanesol and FAK were calculated using "g_hbond" tool with proton donor and acceptor

228 distance $\leq 3.5 \AA$ and the angle between acceptor-donor-hydrogen $\leq 30.0$ degrees.

229

230

231

232

233

234

235

\section{Binding free energy calculations}

Molecular mechanics Poisson-Boltzmann surface area (MMPBSA) (Massova \& Kollman, 2000) approach was to estimate the binding free energy of protein-ligand interaction. For this purpose, "g_mmpbsa" (Kumari, Kumar, \& Lynn, 2014) tool was used. The tool calculates the molecular mechanics potential energy and the free energy of solvation and excludes the entropy calculations. MM-PBSA calculations were performed using 1000 snapshots taken from last $5 \mathrm{~ns}$ of trajectories of the complex system.

\section{Result \& Conclusion}

\section{Docking Analysis:}

\section{Blinding docking and LBCSR Score:}

Solanesol was docked against FAK structure (PDB id: 4Q9S) using Autodock4.2 three times (as mentioned in materials \& methods). For all the three times, a grid of size $126 \times$ $126 \times 126$ with 0.375 A [Figure 1] spacing was created with protein center $($ Centre coordinates $=$ $9.7,0.16,15.1)$ as the grid center, as per the normal "blind docking" protocol. Dielectric constant value was kept default.

Solanesol shows very small cluster with insignificant binding affinity towards FAK when docked "blindly" [Table 1]. Though, the structure show quite high binding affinity towards the kinase but the conformations with higher binding energy fails to form any significant cluster. From all the generated conformations of Solanesol which were having binding energy lower than-4.0 kCal/mol, all the favorable and unfavorable overlaps of the atoms were calculated 
249 using "FindClash" tool of UCSF Chimera. Chimera tool, "FindHbond" was also used to find the

250 hydrogen bonds between the ligand and residue atoms. The default values were kept for all the

251 calculation in Chimera.

252 An in-house python script was used to calculate the number of "Clashes", "Contacts" and

253 "Hbonds" between all the conformations and residues as well as individual scores. The scores

254 from all the three experiments were added to give a final score for each residue [Table 2].

255 Based on the LBCSR score calculated for all three experiments, the scores for all the

256 residues were plotted as graph (Fig 3) as well as plotted as false colour on the 3D structure of the

257 protein (Fig 2). From the LBCSR score it can be inferred that the ligand shows a high affinity

258 towards the region with Asp564, Asn551, Arg550, Leu553 and Ile428, respectively.

Refined docking:

All the residues with more than LBCSR score was considered for the active site

261 prediction. A total of 77 residues were found to be above and incidentally which also forms the

262 ATP binding site and the catalytic loop (546-551) and formed between the N and C lobe [1].

263 Centre of geometry (Coordinates $=10.8,1.0,15.0)$ of these 77 residues were considered

264 for the centre of the binding pocket of Solanesol. A grid of size $60 \times 62 \times 68$ was considered to

265 exactly fit all the 77 residues. Autodock4.2 was again used for docking of Solanesol with FAK

266 with this grid setting for two more times, first time with default setting for 200 conformations

267 and later docked with exhaustive setting for the same number of conformations.

268 All 400 conformations were rescored using DSX online server with CSD settings. (Table 3) 


\section{Analysis of Final Docked structure:}

The hydroxyl end of Solanesol binds to the binding site of ATP and interacts with Ile428,

271 Val436, Ala452, Lys454 and Leu501. These residues which forms the binding pocket for ATP,

272 forms Alkyl-Pi hydrophobic interactions with the double bonds of the ligand. Whereas, the

273 isobutyl end of the ligand, gets attached with the Catalytic loop and $\alpha \mathrm{C}$ helix. The ligand

274 interacts with Phe542, Arg545, Arg550 (Catalytic loop) and Phe478 ( $\alpha \mathrm{C}$ helix terminal) with

275 Alkyl and Sigma-Pi interactions.Pro585, Ile586 near the ATP active site also forms similar

276 hydrophobic interactions with the ligand. Oxygen of Solanesol forms a very conventional H-

277 bond with OE1 of Gln438 which is very near to G-Loop and ATP binding site.[30]

278 These 12 hydrophobic interactions of 11 residues with Solanesol stabilize the ligand at

279 the middle of $\mathrm{N}$ and $\mathrm{C}$ lobe of FAK. As the ligand share interacting residues with both the side it

280 may be act as a better inhibitor for FAK. This structure was used for molecular dynamics studies.

\section{Analysis of Molecular Dynamics}

282 For the measuring the stability, the deviation in the backbone of the protein has been

283 measured relative to time [Fig 5]. After an initial instability, the backbone changes its shapes

284 linearly with a linear slope increase in RMSD between $4.5 \mathrm{~ns}$ to $7.7 \mathrm{~ns}$ after again a short

285 destabilization the system vaguely equilibrates from 9.4 ns to $14.2 \mathrm{~ns}$. It takes the system almost

$28615 \mathrm{~ns}$ to equilibrate, after which the system maintains it position and shape. [Fig 5]

287 Comparison of the RMSD of both the trajectories from the minimized structures shows,

288 Solanesol bound FAK backbone show more stability than that of independent FAK backbone.

289 RMSF (Root mean Square Fluctuation) of Solanesol bond FAK exhibits less fluctuation at the

290 places where Solanesol is bond [Fig 6]. 
292 analyzing the change in surface area with respect to time. It show a quite negative correlation

293 with the RMSD suggesting the better binding of the ligand leads to a lower surface area of the

294 protein thus closing the active site for any further contact. It remains stable for the wider part of

295 6ns -18ns range after which the backbone gets stabilized and may also affect the surface area.

298 Binding site residues for Solanesol were selected by taking 3.5A radius from Solanesol.

299 Molecular mechanics Poisson-Boltzmann surface area (MM-PBSA) was calculated for the last 5

300 ns with 20 ps steps for the binding site residues versus Solanesol using g_mmpbsa tool.

301 Per residue analysis of the result was done and plotted using the script provided. This analysis

302 suggests that, Ile428, Gly431, Val436, Val484, Met499, Cys502 and Leu553 interacts most

303 favorably with the ligand. Interestingly these all residues are part of ATP binding pocket of

304 FAK. Gly431 is a part of the G-loop which helps in the phosphorylation of the protein also

305 interacts favorably along with Leu584 which is part of the activation loop.

306 MM-PBSA based binding affinity $(\Delta \Delta \mathrm{G})$ was calculated using the g_mmpbsa provided script.

$$
\Delta \mathrm{G}_{\mathrm{Sol}}=\Delta \mathrm{G}_{\mathrm{PB}}+\Delta \mathrm{G}_{\mathrm{SA}}
$$


$311 \Delta \mathrm{E}_{\mathrm{MM}}, \Delta \mathrm{G}_{\mathrm{Sol}}$ and $\mathrm{T} \Delta \mathrm{S}$ represent the molecular mechanics component in the gas phase,

312 stabilization energy due to salvation, and a vibrational entropy term, respectively. $\Delta \mathrm{E}_{\mathrm{MM}}$ is the

313 summation of $\Delta \mathrm{E}_{\mathrm{int}}, \Delta \mathrm{E}_{\mathrm{col}}$, and $\Delta \mathrm{E}_{\mathrm{vdw}}$, which are the internal, coulomb, and van der Waals

314 interaction terms, respectively. $\Delta \mathrm{G}_{\mathrm{sol}}$ is the salvation energy and it is divided into an electrostatic

315 salvation free energy $\left(\mathrm{G}_{\mathrm{PB}}\right)$ and a non-polar salvation free energy $\left(\mathrm{G}_{\mathrm{SA}}\right)$.

316 A very low $\Delta \Delta \mathrm{G}$, of $-113.85 \mathrm{~kJ} / \mathrm{Mol}$, (Table 4) proves Solanesol have a very high binding

317 affinity towards the FAK structure. The change in binding energy with time was also plotted.

318 [Fig 7], It shows that the ligand gets stabilized with a very high binding affinity after 17ns of

319 simulation.

\section{Conclusion}

322 In this study, we have established a new method for scoring of binding pocket residues

323 based on blind docking methodology of highly flexible residue. Knowledge based scoring

324 function, MD simulation and binding free-energy calculations were performed to investigate and

325 validate the binding conformation of Solanesol on Focal adhesion kinase at the molecular level

326 found by LBCSR. This scoring was crucial as blind docking fails to give high confidence data

327 for binding of highly flexible residues.

Based on the proposed LBCSR score, residues were identified which give high contact

330 score with ligand in multiple blind docking instances. Refined docking was used to identify the

331 best pose for this site. Knowledge based scoring function, DSX, was used to validate the docking

332 result on which 20ns MD simulation was performed. Simulation of only protein structure of

333 FAK protein was performed for establishing a control for MD simulations. 
The MM-PBSA binding free-energy calculations further confirmed the results of the MD simulation. This analysis, established a correlation between the LBCSR and favorably interacting residues. It is in a good agreement with experimental results which shows Solanesol binds at ATP binding site and inhibit the phosphorylation of Focal Adhesion Kinase. This study may help in further analysis and understanding of flexible compounds and inhibition of FAK using the same.

\section{Reference}

343 Cohen, L. A., \& Guan, J. L. (2005). Mechanisms of focal adhesion kinase regulation.

$344 \quad$ Current cancer drug targets, 5(8), 629-643. Ewald sums in large systems. The Journal of chemical physics, 98(12), 10089-10092. 09, Gaussian. Inc, Wallingford, CT. selective and orally bioavailable protein kinase $\mathrm{C} \theta(\mathrm{PKC} \theta)$ inhibitors from a fragment hit. Journal of medicinal chemistry, 58(1), 222-236. journal of biochemistry \& cell biology, 29(8), 1085-1096. 
Hanahan, D., \& Weinberg, R. A. (2011). Hallmarks of cancer: the next generation. cell, $144(5), 646-674$.

Hetényi, C., \& van der Spoel, D. (2002). Efficient docking of peptides to proteins without prior knowledge of the binding site. Protein science, 11(7), 1729-1737.

Hetényi, C., \& van der Spoel, D. (2006). Blind docking of drug-sized compounds to proteins with up to a thousand residues. FEBS letters, 580(5), 1447-1450. for highly efficient, load-balanced, and scalable molecular simulation. Journal of chemical theory and computation, 4(3), 435-447. Shoemaker, B.A. and Wang, J., 2015. PubChem substance and compound databases. Nucleic acids research, gkv951. 1951-1962. 
Massova, I., \& Kollman, P. A. (2000). Combined molecular mechanical and continuum solvent approach (MM-PBSA/GBSA) to predict ligand binding. Perspectives in drug discovery and design, 18(1), 113-135. Olson, A. J. (2009). AutoDock4 and AutoDockTools4: Automated docking with selective receptor flexibility. Journal of computational chemistry, 30(16), 2785-2791.

National Center for Biotechnology Information (NCBI). PubChem Compound Database; $\mathrm{CID}=5477212$, https://pubchem.ncbi.nlm.nih.gov/compound/5477212 (accessed Aug. 11, 2016). assessment of protein-ligand complexes. Journal of chemical information and modeling, 51(10), 2731-2745.

Okamoto, Y., Tsuji, M., \& Yamazaki, H. (1994). U.S. Patent No. 5,306,714. Washington, DC: U.S. Patent and Trademark Office.

Pettersen, E. F., Goddard, T. D., Huang, C. C., Couch, G. S., Greenblatt, D. M., Meng, E. C., \& Ferrin, T. E. (2004). UCSF Chimera-a visualization system for exploratory research and analysis. Journal of computational chemistry, 25(13), 1605-1612. synthesis of [3-14C] solanesol. Journal of Labelled Compounds and Radiopharmaceuticals, 56(9-10), 485-491. 
Srivastava, S., Raj, K., Khare, P., Bhaduri, A.P., Chander, R., Raghubir, R., Mahendra, K., Rao, C.N. and Prabhu, S.K. (2009). Novel hybrid natural products derived from solanesol as wound healing agents. Indian journal of chemistry. Section B, Organic including medicinal, 48(2), 237.

Potentiation of Antitumor Drugs against Multidrug-resistant and Sensitive Cells in vitro. Japanese journal of cancer research, 81(3), 298-303.

Tomida, A., \& Suzuki, H. (1990). Synergistic Effect in Culture of Bleomycin-group Isoprenoid. Japanese journal of cancer research, 81(11), 1184-1190. (1996). PRODRG, a program for generating molecular topologies and unique molecular descriptors from coordinates of small molecules. Journal of computer-aided molecular design, 10(3), 255-262. in cancer therapy. Biochemical pharmacology, 73(5), 597-609. Reviews, 14(3), 403-417. 


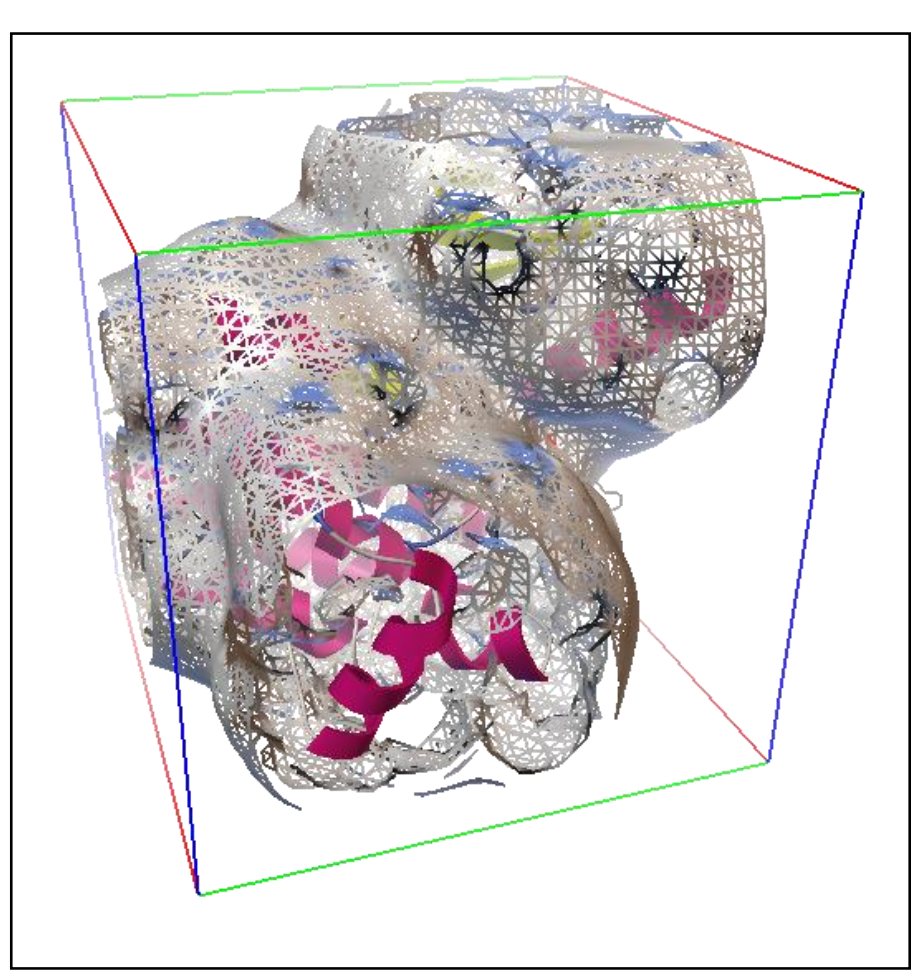

$1=0$

4Jง

455 Figure 1: Autogrid map on Focal adhesion kinase as generated by Autogrid4 when blind docking 456 is done on the whole surface. 

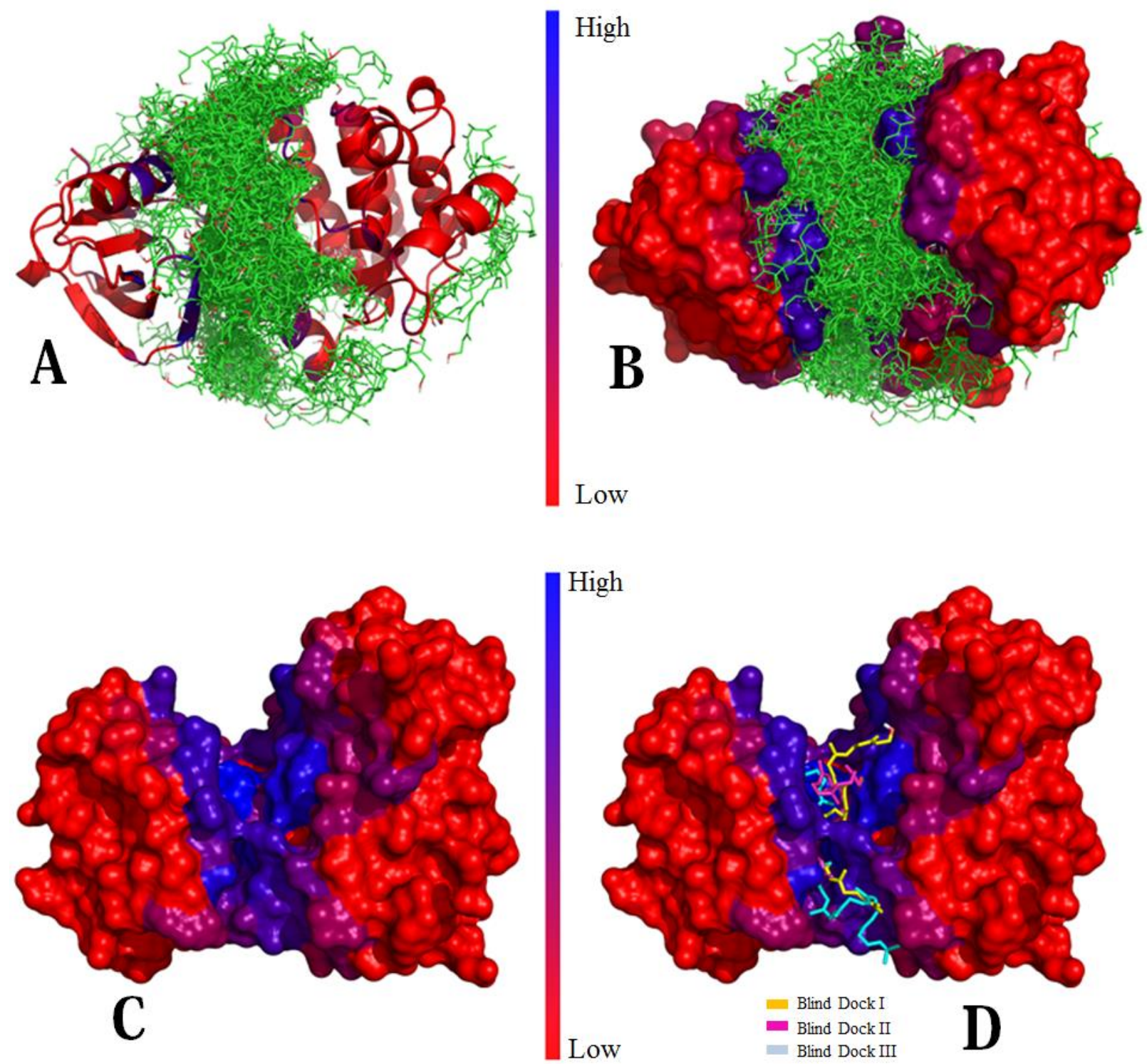

459

460

Figure 2: (A,B)FAK structure with colour based on LBCSR score show all the conformations docked "blindly". (C)

462 structures (based on binding energy) from the three docking experiments. 


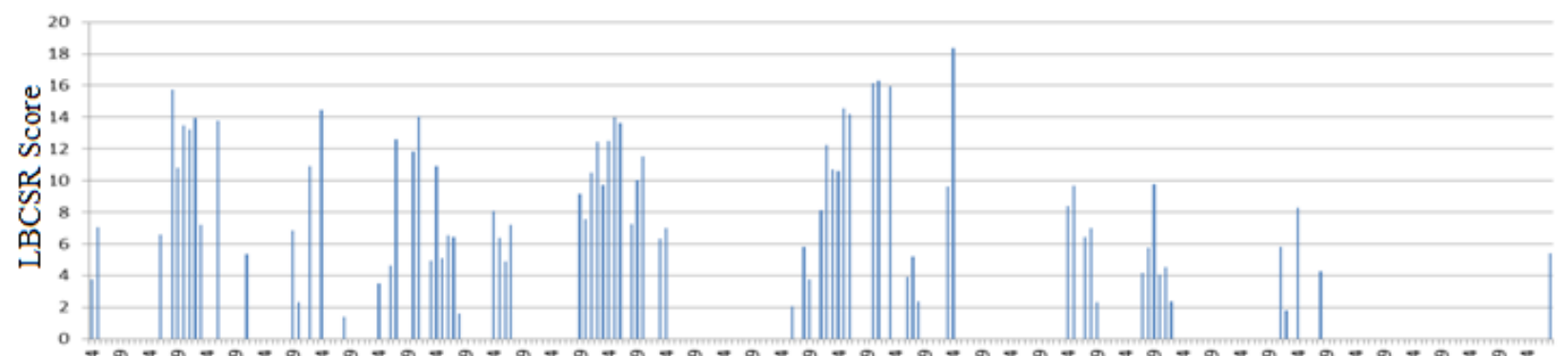

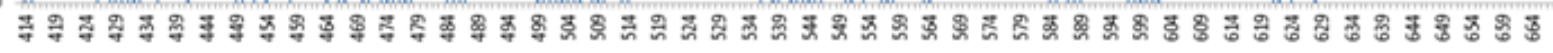

Figure 3: Calculated average LBCSR scores for all the residues of Focal Adhesion Kinase for

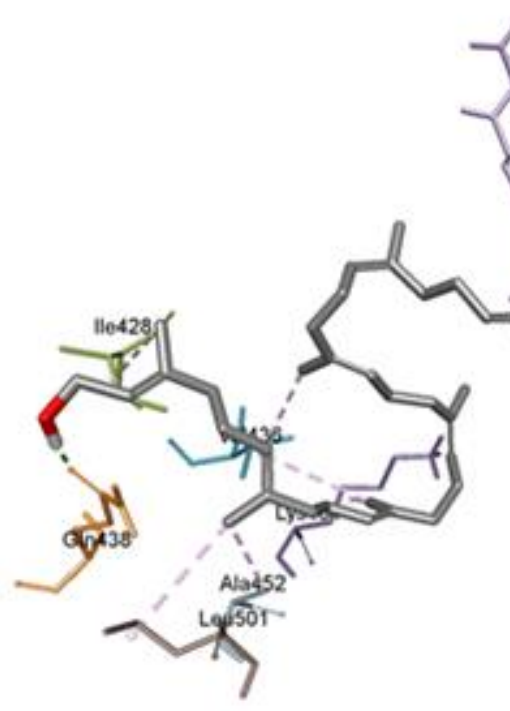

471

472

473

474

Figure 4: 3D (A) and 2D (B) mapof Solanesol when bound to Focal adhesion kinase (FAK)

475

\section{Residue Number}




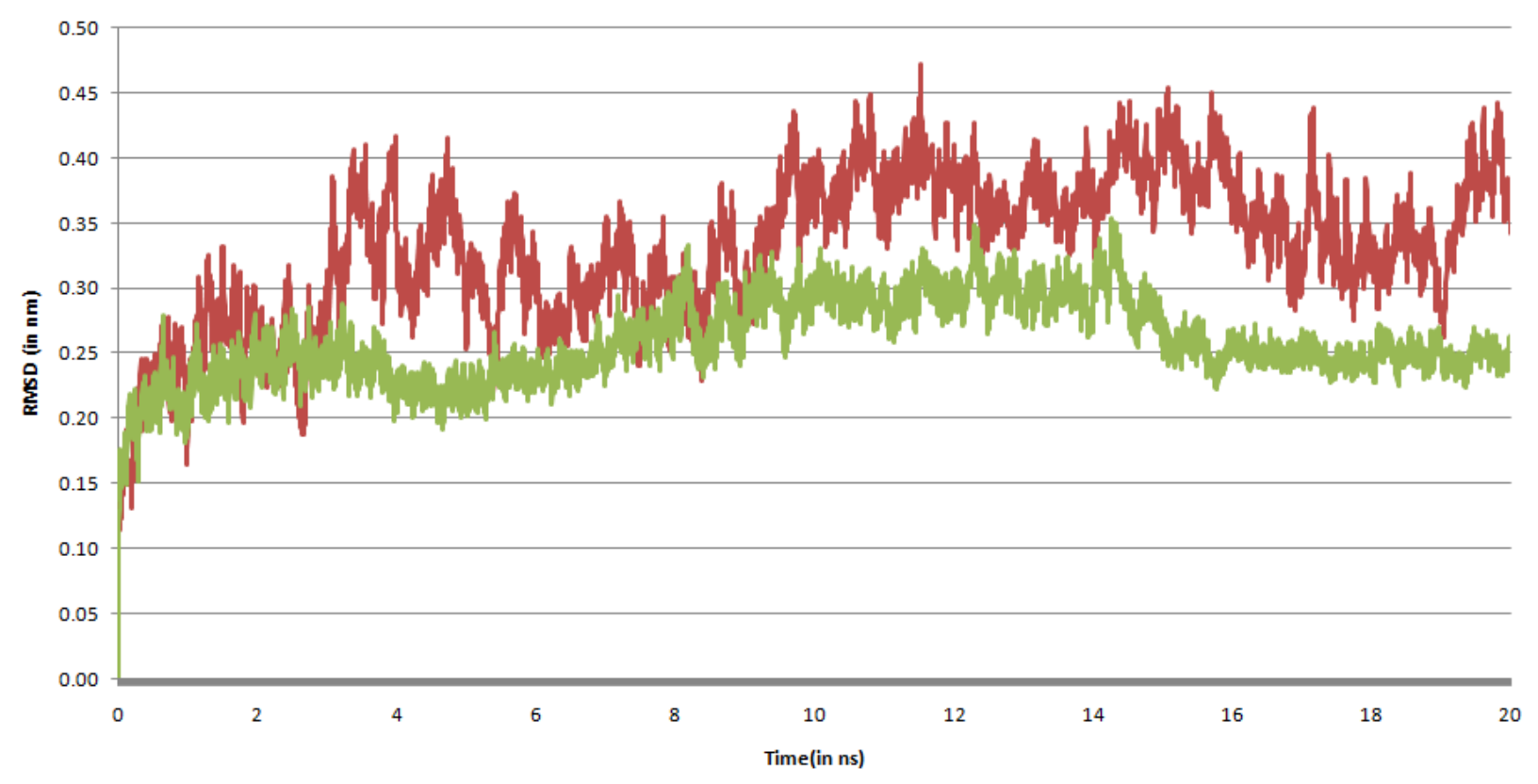

Figure 5: Root mean square deviation (RMSD) of Focal adhesion kinase backbone atoms when bound to Solanesol. It gets stabilized nearly after $16 \mathrm{~ns}$ of simulation.

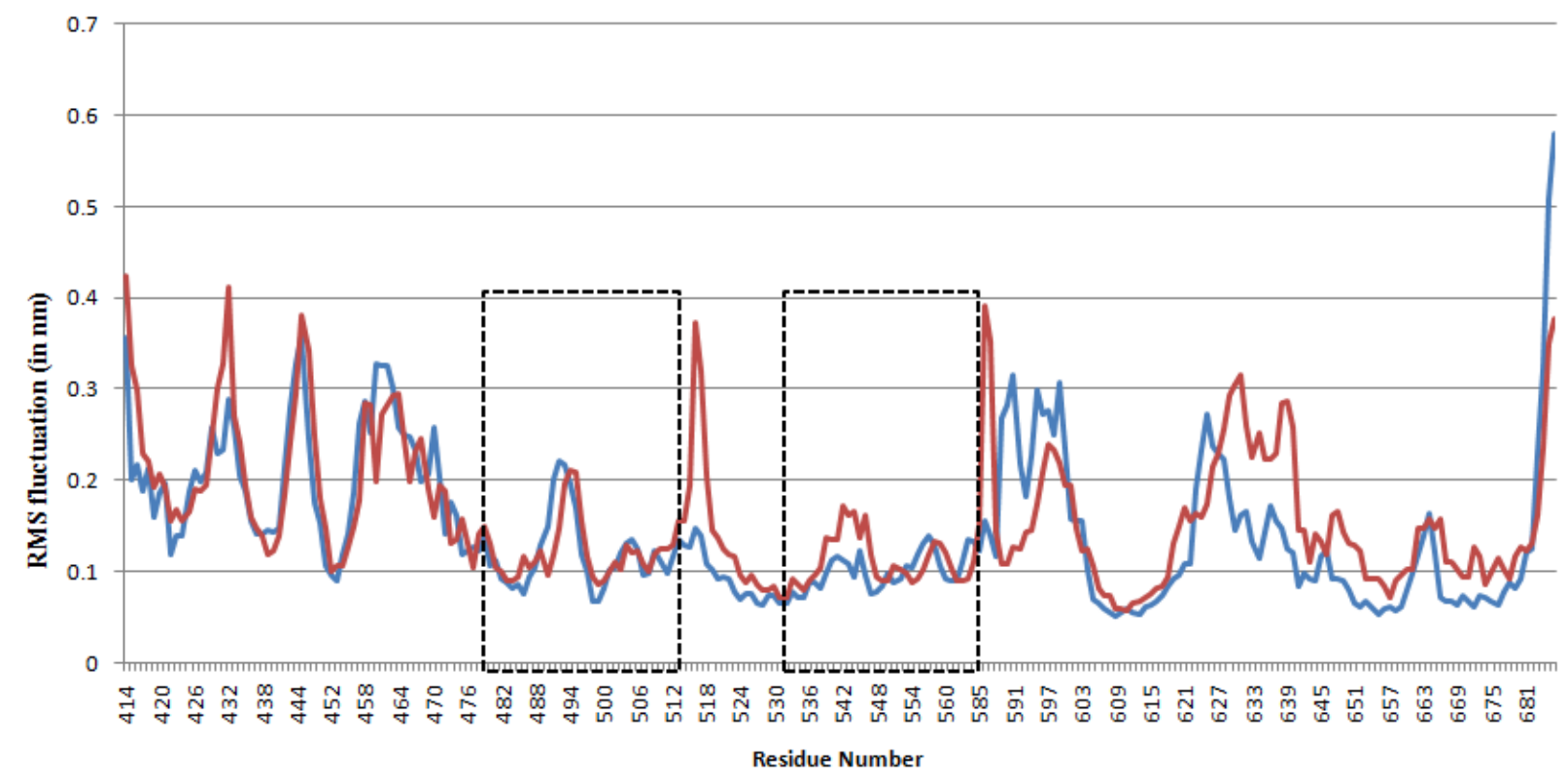

480 Figure 6: RMS Fluctuation (in $\mathrm{nm}$ ) in alpha carbon atom of each residue in FAK (Red) and

481 Solanesol bound FAK (Blue). Boxes show the binding site region of FAK. 


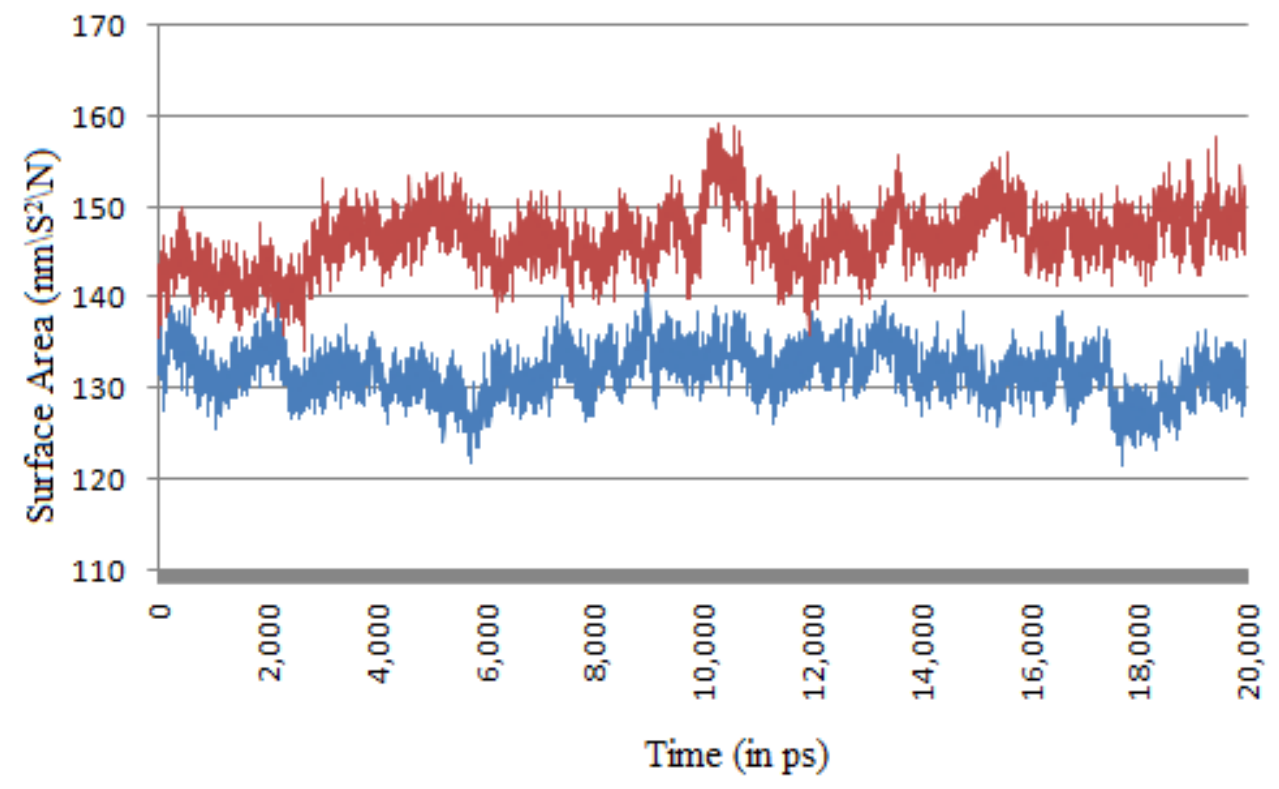

483 Figure 7: Total Solvant Accessible Surface Area of Focal Adhesion Kinase with (Blue) and 484 without (Red) bound Solanesol.

485

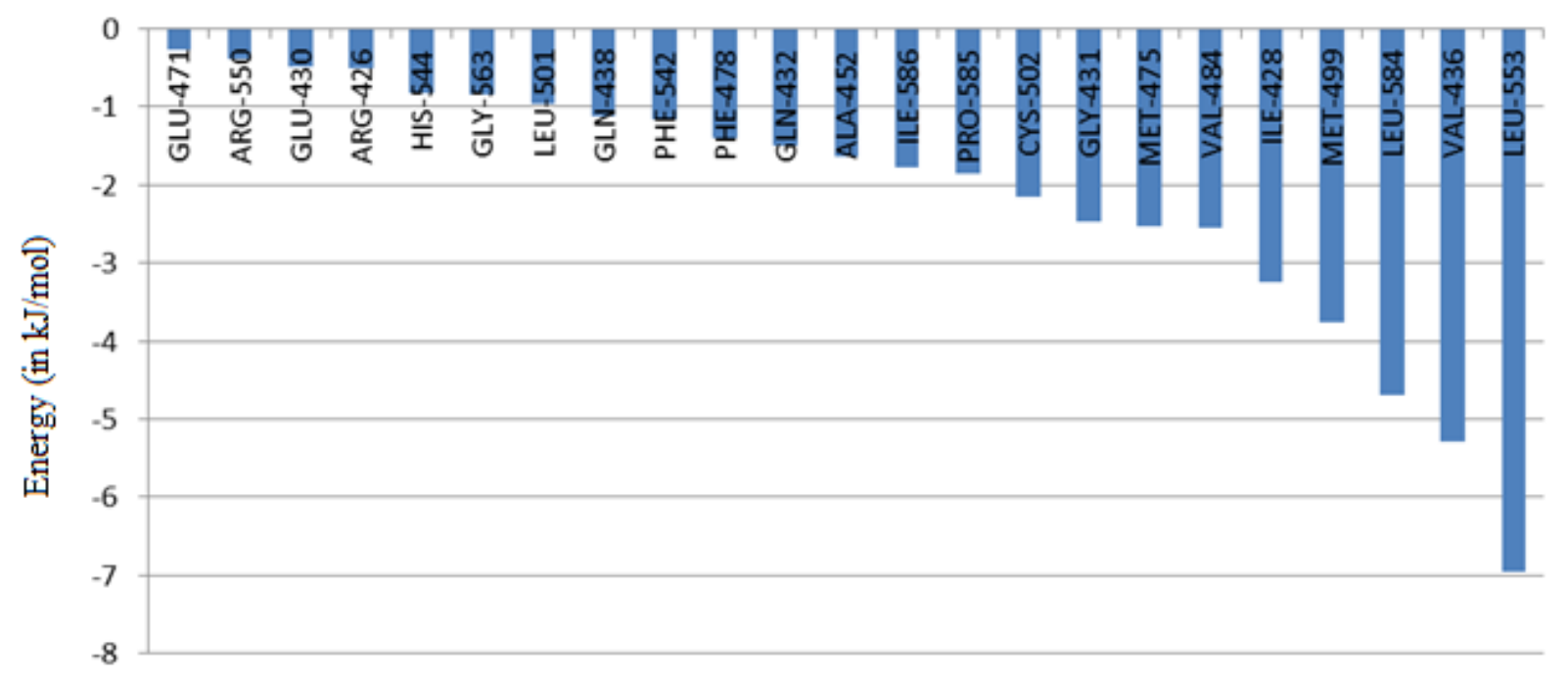

487 Figure 8: MM-PBSA based residue energy profile for active site residues. 


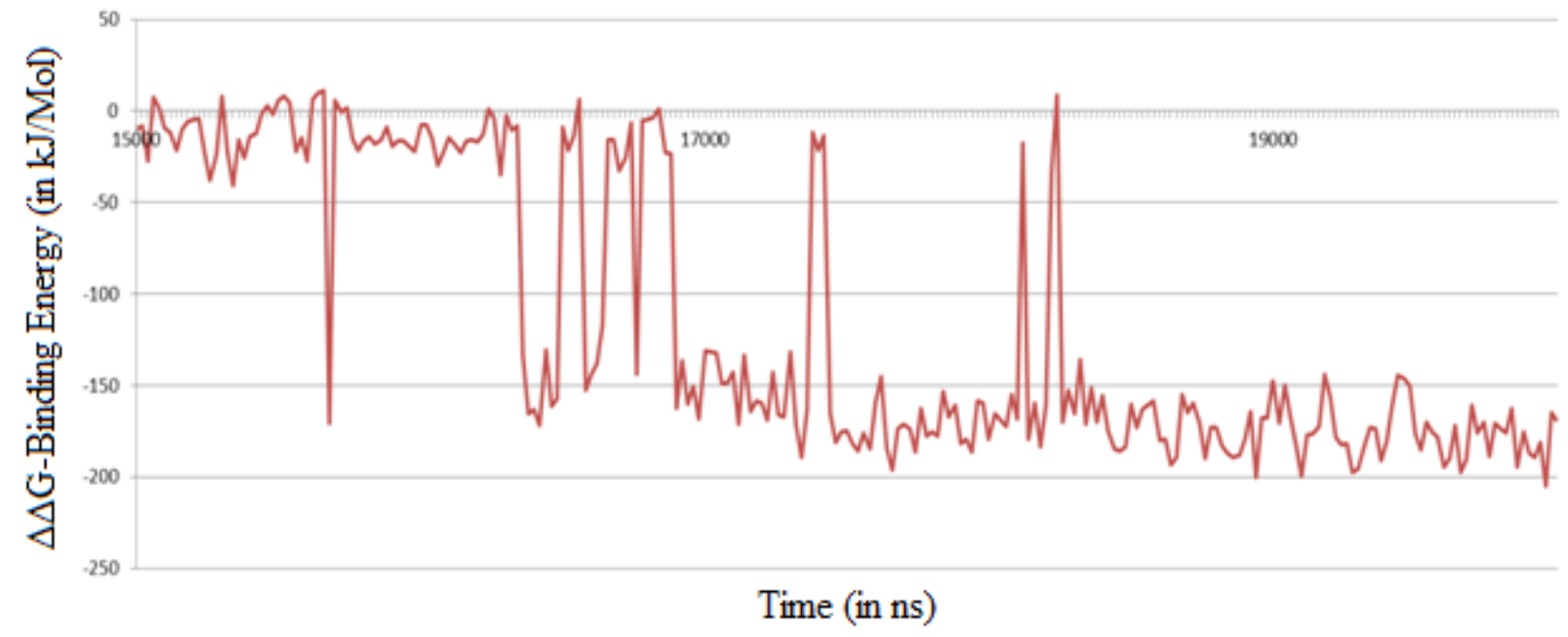

490 Figure 9: Binding Energy $(\Delta \Delta \mathrm{G})[\mathrm{kJ} / \mathrm{mol}])$ using MM-PBSA method w.r.t time.

491

492

\begin{tabular}{ccccccc}
\hline Experiment & $\begin{array}{c}\text { Total } \\
\text { Conformations }\end{array}$ & Grid Center & $\begin{array}{c}\text { L.B.E } \\
(\mathbf{\Delta} \mathbf{G}) \text { in } \\
\mathbf{k C a l} / \mathbf{M o l}\end{array}$ & $\begin{array}{c}\text { Total } \\
\text { Cluster }\end{array}$ & $\begin{array}{c}\text { Highest } \\
\text { number of } \\
\text { member in } \\
\text { any cluster }\end{array}$ & $\begin{array}{c}\text { Average B.E of } \\
\text { cluster with } \\
\text { most number } \\
\text { of members }\end{array}$ \\
\hline Blind Docking I & 500 & Center of Protein & -7.08 & 328 & 10 & 0.23 \\
Blind Docking II & 200 & Center of Protein & -8.54 & 160 & 5 & -0.19 \\
Blind Docking III & 200 & Center of Protein & -5.68 & 187 & 3 & 0.13 \\
\hline
\end{tabular}

493 Table 1: Blind docking analysis with three different experiments (with different starting

494 conformations) using standard Autodock protocol

495

496

\begin{tabular}{cccc}
\hline Residues & Blind Docking I & Blind Docking II & Blind Docking III \\
\hline LYS-454 & 5.66 & 16.50 & 11.27 \\
ASP-564 & 8.89 & 22.35 & 14.77 \\
ARG-550 & 7.48 & 16.33 & 13.64 \\
GLU-500 & 2.48 & 12.06 & 6.14 \\
ARG-426 & 3.04 & 12.11 & 3.18 \\
GLU-430 & 5.02 & 13.87 & 13.49 \\
LEU-501 & 4.25 & 11.98 & 8.23 \\
GLY-563 & 4.25 & 12.08 & 6.76
\end{tabular}




$\begin{array}{cccc}\text { GLU-471 } & 6.78 & 22.50 & 9.55 \\ \text { GLN-432 } & 5.65 & 21.14 & 11.85 \\ \text { ALA-452 } & 4.88 & 12.30 & 7.36 \\ \text { CYS-502 } & 5.31 & 12.32 & 9.13 \\ \text { PRO-585 } & 4.09 & 15.50 & 7.17 \\ \text { HIS-544 } & 4.98 & 15.97 & 9.29 \\ \text { GLY-431 } & 5.12 & 17.82 & 11.71 \\ \text { VAL-484 } & 3.74 & 8.93 & 5.78 \\ \text { MET-475 } & 2.08 & 10.95 & 0.69 \\ \text { ILE-428 } & 5.79 & 16.92 & 13.77 \\ \text { MET-499 } & 4.42 & 10.30 & 5.78 \\ \text { LEU-584 } & 2.30 & 13.20 & 10.42 \\ \text { VAL-436 } & 5.55 & 15.14 & 11.36 \\ \text { LEU-553 } & 6.30 & 17.18 & 13.47\end{array}$

Table 2: Top residues with best LBCSR scores of all three experiments

\begin{tabular}{cccccc}
\hline & Total Score & $\begin{array}{c}\text { Per Contact } \\
\text { Score }\end{array}$ & Torsional Score & SAS Score & $\begin{array}{c}\text { Binding Free } \\
\text { Energy }\end{array}$ \\
\hline Exhaustive Parameter & -166.61 & -0.19 & -11.13 & -16.10 & -7.30 \\
Default Parameter & -131.69 & -0.17 & -12.43 & -8.59 & -7.25 \\
\hline
\end{tabular}

499 Table 3: DSX, knowledge based scoring of Exhaustive and normal Docking

500

\begin{tabular}{ccccc}
\hline $\begin{array}{c}\Delta \text { G-vdw (in } \\
\mathrm{kJ} / \mathrm{Mol})\end{array}$ & $\begin{array}{c}\Delta \text { G-electro (in } \\
\mathrm{kJ} / \mathrm{Mol})\end{array}$ & $\begin{array}{c}\Delta \mathrm{G}-\text { polar (in } \\
\mathrm{kJ} / \mathrm{Mol})\end{array}$ & $\begin{array}{c}\Delta \mathrm{G}-\mathrm{SAS} \text { (in } \\
\mathrm{kJ} / \mathrm{Mol})\end{array}$ & $\begin{array}{c}\boldsymbol{\Delta} \boldsymbol{\Delta G} \text {-BE (in } \\
\mathbf{k J} / \mathbf{M o l})\end{array}$ \\
\hline-145.35 & -1.71 & 52.05 & -18.85 & $\mathbf{- 1 1 3 . 8 5}$ \\
& & & & \\
\hline
\end{tabular}

501 Table 4: MM-PBSA based final Binding free energy of Solanesol with Focal Adhesion Kinase 502 